Pacific Journal of Mathematics

CLOPEN REALCOMPACTIFICATION OF A MAPPING 


\title{
CLOPEN REALCOMPACTIFICATION OF A MAPPING
}

\section{TAKESI ISIWATA}

\begin{abstract}
In this note, we give a necessary and sufficient condition on $\varphi$ : $X \rightarrow Y$ for $v \varphi$ to be an open perfect mapping of $v X$ onto $v Y$ and other related results.
\end{abstract}

Throughout this paper, by a space we mean a completely regular Hausdorff space and mappings are continuous and we assume familiarity with [1] whose notation and terminology will be used throughout. We denote by $\varphi: X \rightarrow Y$ a map of $X$ onto $Y$, by $\beta X(v X)$ the Stone-Čech compactification (Hewitt realcompactification) of $X$ and by $\beta \varphi(v \varphi=$ $(\beta \varphi) \mid v X)$ the Stone extension (realcompactification) over $\beta X(v X)$ of $\varphi$.

Concerning clopenness of $v \varphi$ of a clopen map $\varphi: X \rightarrow Y$ the following results are known.

THEOREM A (Ishii [4]). If $\varphi: X \rightarrow Y$ is an open quasi-perfect map, then $v \varphi$ is an open perfect map of $v X$ onto $v Y$.

THEOREM B (Morita [8]). If $\varphi: X \rightarrow Y$ is a clopen map such that the boundary of each fiber is relatively pseudocompact, then $v \varphi$ is also a clopen map of $v X$ onto $v Y$.

In $\$ 2$, concerning Theorem $\mathrm{A}$ we give a necessary and sufficient condition on $\varphi$ for $v \varphi$ to be an open perfect map of $v X$ onto $v Y$ without using the theory of hyper-spaces (Theorem 2.3 below) and a necessary and sufficient condition on $\varphi$ for $v \varphi$ to be an open $R C$-preserving map of $v X$ onto $v Y$ under some condition (Theorem 2.6 below).

We use the following notation and abbreviation: $C(X)$ is the set of real-valued continuous functions defined on $X, C(X ; \varphi)=\{f \in C(X) ; f$ is $\varphi$-bounded $\}, \operatorname{Bd} A=$ the boundary of $A$, usc = upper semicontinuous, lsc $=$ lower semicontinuous and $\omega\left(\omega_{1}\right)=$ the first infinite (uncountabel) ordinal, clopen $=$ closed and open.

\section{Definitions and Lemmas.}

1.1. Definition. Let $\varphi: X \rightarrow Y . f \in C(X)$ is said to be $\varphi$-bounded if $\sup \left\{|f(x)| ; x \in \varphi^{-1}(y)\right\}<\infty$ for every $y \in Y$. Whenever $f$ is $\varphi$-bounded, 
we put

$$
\begin{aligned}
& f^{s}(y)=\sup \left\{f(x) ; x \in \varphi^{-1}(y)\right\} \quad \text { and } \\
& f^{i}(y)=\inf \left\{f(x) ; x \in \varphi^{-1}(y)\right\} \quad \text { for each } y \in Y .
\end{aligned}
$$

A subset $A$ of $X$ is relatively pseudocompact if $f \mid A$ is bounded for each $f \in C(X) . \varphi: X \rightarrow Y$ is said to be

(1) $W Z$ if $\operatorname{cl}_{\beta X} \varphi^{-1} y=(\beta \varphi)^{-1} y$ for each $y \in Y$ [5].

(2) $W_{r} N$ if $\mathrm{cl}_{\beta X} \varphi^{-1} R=(\beta \varphi)^{-1}\left(\mathrm{cl}_{\beta Y} R\right)$ for every regular closed set $R$ of $Y[3]$.

(3) *-open $\left(W^{*}\right.$-open $)$ if $\operatorname{int}(\operatorname{cl} \varphi U) \supset \varphi U(\operatorname{int}(\operatorname{cl} \varphi U) \neq \varnothing)$ for every open set $U$ of $X[2,7]$.

(4) $\beta$-open if $\varphi$ is *-open and $W_{r} N$.

(5) a $d^{*}$-map if $\cap \operatorname{cl} \varphi Z_{n}=\varnothing$ for any decreasing sequence $\left\{Z_{n}\right\}$ of zero sets of $X$ with empty intersection [6].

(6) $R C$-preserving (an $R C$-map) if $\varphi R$ is regular closed(closed) for every regular closed set $R$ of $X$ [2].

We note that (1) a closed map is a $Z$-map and a $Z$-map is $W Z$ [5], (2) an open map is *-open and a ${ }^{*}$-open map is $W^{*}$-open [7], (3) a space $Y$ is $\mathrm{cb} *$ iff any $d^{*}$-map onto $Y$ is hyper-real, i.e., $v \varphi$ is a perfect map onto $v Y$ [6], (4) an $R C$-preserving map is $R C$ and (5) an open $W Z$-map is $\beta$-open by $1.2(1,5)$ below. Thus it is easy to see that if $\varphi$ is $\beta$-open, then $(\beta \varphi) \mid Z$ : $Z \rightarrow(\beta \varphi) Z$ is $\beta$-open for each $Z$ with $X \subset Z \subset \beta X$. $Y \supset B$ is said to be $\varphi-d^{*}$ if $(\beta \varphi)^{-1} B \subset v X$. By 1.2(4) below, $\varphi$ is a $d^{*}$-map iff $Y$ is $\varphi-d^{*}$.

LEMMA 1.2. Let $\varphi: X \rightarrow Y$.

(1) If $\varphi$ is $W Z$, then $\varphi$ is open iff $\beta \varphi$ is open [5].

(2) if $\varphi$ is open (WZ), then $f^{\prime}$ is usc (lsc) and $f^{s}$ is lsc (usc) for every $f \in C(X: \varphi)$ (for example, see [5]).

(3) If $\varphi$ is open $W Z$, then $f^{i}$ and $f^{s} \in C(Y)$ for every $f \in C(X ; \varphi)$ [5].

(4) $\varphi$ is a d $d^{*}$-map iff $(\beta \varphi)^{-1} Y \subset v X[6]$.

(5) $\varphi$ is $\beta$-open iff $\beta \varphi$ is open [7].

(6) If $\varphi$ is an $R C$-map, then $\varphi$ is $W Z$ [3].

(7) $\varphi$ is $R C$-preserving iff $\varphi$ is a $W^{*}$-open $R C$-map [2].

\section{Main Theorems.}

Lemma 2.1. Let $\varphi: X \rightarrow Y$. Then the following are equivalent:

(1) $\varphi$ is $W Z$ (open).

(2) $f^{i}$ is lsc (usc) for every $f \in C(X ; \varphi)$

(3) $f^{s}$ is usc (lsc) for every $f \in C(X ; \varphi)$. 
Proof. (2) $\Leftrightarrow$ (3) is evident. (1) $\Rightarrow(2)$. From 1.2(2).

We will prove $(2) \Rightarrow(1)$. Suppose that $\varphi$ is not $W Z$. Then there are $y \in Y$ and $p \in \beta X$ with $p \in(\beta \varphi)^{-1} y-\operatorname{cl}_{\beta X} \varphi^{-1} y$. Since $p \notin \operatorname{cl}_{\beta X} \varphi^{-1} y$, there is $g \in C(\beta X)$ such that $p \in \operatorname{int}_{\beta X} Z(g)$ and $g=1$ on $\operatorname{cl}_{\beta X} \varphi^{-1} y$. Let us put $f=g \mid X$. Then $f \in C(X), f^{i}(y)=1, A=Z(f) \neq \varnothing$ and $p \in$ $\mathrm{cl}_{\beta X} A$. On the other hand, $\operatorname{cl}_{\beta Y} \varphi A=\operatorname{cl}_{\beta Y}(\beta \varphi) A=(\beta \varphi) \mathrm{cl}_{\beta X} A \ni(\beta \varphi) p$ $=y$. This shows $y \in \operatorname{cl} \varphi A$ and hence for each neighborhood $V$ of $y$, there is $z \in V$ with $f^{i}(z)=0$, i.e., $f^{i}$ is not lsc.

Now suppose that $\varphi$ is not open. Then there are a point $x$ and an open set $U \ni x$ such that $V-\varphi U \neq \varnothing$ for every open set $V \ni y=\varphi(x)$. Let $f \in C(X ; \varphi)$ such that $x \in \operatorname{int} Z(f) \subset U$ and $f=1$ on $X-U$. Obviously $f^{i}(y)=0$ and $f^{i}=1$ on $V-\varphi U$. This shows that $f^{i}$ is not usc.

Using 2.1 , it is easy to see the following:

THEOREM 2.2. $\varphi: X \rightarrow Y$ is open $W Z$ iff $f^{i}$ and $f^{s} \in C(Y)$ for every $f \in C(X ; \varphi)$ equivalently,

$$
C(Y)=\left\{f^{i} ; f \in C(X ; \varphi)\right\}=\left\{f^{s} ; f \in C(X ; \varphi)\right\} .
$$

THEOREM 2.3. $\varphi: X \rightarrow Y$ is a $\beta$-open $d^{*}$-map iff $v \varphi$ is an open perfect map of $v X$ onto $v Y$.

Proof. $\Leftarrow)$ From $1.2(1,4,5)$ and $\left.(\beta \varphi)^{-1} Y \subset(\beta \varphi)^{-1} v Y=v X . \Rightarrow\right)$ By $1.2(5), \beta \varphi$ is open. We will prove that $v \varphi$ is a perfect map onto $v Y$. To do this, it suffices to show that $(\beta \varphi) p=q \in \beta Y-v Y$ for every $p \in \beta X-$ $v X$. Let $p \in \beta X-v X$. Then there is $f \in C(\beta X)$ with $p \in Z(f) \subset \beta X-$ $v X . \beta \varphi$ being open $W Z$ by $1.2(5)$, it follows from 2.2 that $f^{i} \in C(\beta Y)$, $f^{i}(q)=0$ and $f^{i}>0$ on $Y$. This shows $q \in \beta Y-v Y$, so $v \varphi$ is a perfect map onto $v Y$. Since $\beta(v \varphi)=\beta \varphi$ and $\beta \varphi$ is open, $v \varphi$ is open by $1.2(1)$. Thus $v \varphi$ is an open perfect map of $v X$ onto $v Y$.

2.4. EXAmple. Let $X=\left[0, \omega_{1}\right]^{2}-\left\{\left(\omega_{1}, \alpha\right) ; \omega \leq \alpha \leq \omega_{1}\right\}, Y=\left[0, \omega_{1}\right]$ and $\varphi$ the projection of $X$ onto $Y$. It is obvious that $\varphi$ is not $W Z$ and hence not closed and $\varphi^{-1}\left(\omega_{1}\right)$ is not compact. On the other hand $\beta \varphi$ : $\beta X=v X=\left[0, \omega_{1}\right]^{2} \rightarrow Y=v Y=\beta Y$ is open perfect (compare with the assumption of Theorem $\mathrm{A}$ ).

\subsection{LEMMA. If $\varphi: X \rightarrow Y$ is $a *$-open $R C$-map, then $\varphi$ is open.}

Proof. Let $U$ be open in $X$ and $x \in U$. Take a regular closed set $R$ with $x \in$ int $R \subset R \subset U$. Since $\varphi$ is a $*$-open $R C$-map, we have $y=\varphi(x)$ $\in \operatorname{int}(\operatorname{cl} \varphi($ int $R)) \subset \varphi R \subset \varphi U$, so $y \in$ int $\varphi U$. Thus $\varphi$ is open. 
In the following we put

$$
\begin{aligned}
& Y_{d}=\left\{y \in Y ; \varphi^{-1} y \text { is open but not relatively pseudocompact }\right\}, \\
& Y_{e}=X-Y_{d} .
\end{aligned}
$$

THEOREM 2.6. $\varphi: X \rightarrow Y$ is a $\beta$-open map such that $Y_{e}$ is $\varphi$ - $d^{*}$ iff $v \varphi$ is an open $R C$-preserving map of $v X$ onto $v Y$ such that $\mathrm{cl}_{v Y} Y_{e}$ is $(v \varphi)-d^{*}$.

Proof. $\Leftarrow)$ Since $v \varphi$ is open $W Z$ by $1.2(6,7), \beta \varphi$ is open by $1.2(1)$ and $\varphi$ is a $\beta$-open map by 1.2(5). The fact that $\operatorname{cl}_{v Y} Y_{e}$ is $(v \varphi)-d^{*}$ implies that $Y_{e}$ is $\varphi-d^{*}$.

$\Rightarrow$ ) (1) We will first prove that if $p \in \beta X-v X$ and $(\beta \varphi) p=q \in v Y$, then there is a clopen subset $D$ of $Y$ such that $q \in \operatorname{cl}_{v Y} D, D \subset Y_{d}$ and $\operatorname{cl}_{v Y} D \cap \operatorname{cl}_{v Y} Y_{e}=\varnothing$. There is $f \in C(\beta X)$ with $p \in Z(f) \subset \beta X-v X$. By $1.2(5), \beta \varphi$ is open. Thus $f^{i} \in C(\beta Y)$. Since $Y_{e}$ is $\varphi-d^{*}, f^{i}>0$ on $Y_{e}$ and hence $Z\left(f^{i}\right) \cap Y_{e}=\varnothing$. Since $f^{i}(q)=0, q \in v Y$ and $Z\left(f^{i}\right)$ is closed. $D=Z\left(f^{i}\right) \cap Y_{d}=Z\left(f^{i}\right) \cap Y$ is a non-empty clopen discrete subset of $Y$ contained in $Y_{d} \cdot \mathrm{Cl}_{v Y} D=Z\left(f^{i}\right) \cap v Y$ implies $q \in \operatorname{cl}_{v Y} D$ and $\operatorname{cl}_{v Y} D \cap$ $\mathrm{cl}_{v Y} Y_{e}=\varnothing$.

(2) Let us put $\mathscr{D}=\left\{D \subset Y_{d} ; D\right.$ is a clopen subset of $\left.Y\right\}$ and $\operatorname{cl}_{v Y} \mathscr{D}=\bigcup\left\{\operatorname{cl}_{v Y} D ; D \in \mathscr{D}\right\}$. Then it is easy to see the following

$$
v Y=\operatorname{cl}_{v Y} \mathscr{D} \cup \operatorname{cl}_{v Y} Y_{e}, \quad \operatorname{cl}_{v Y} \mathscr{D} \cap \operatorname{cl}_{v Y} Y_{e}=\varnothing
$$

and

$$
(\beta \varphi)^{-1} \mathrm{cl}_{v Y} Y_{e} \subset v X .
$$

(3) $v \varphi$ is onto $v Y$. Let $q \in \operatorname{cl}_{v Y} D, D \in \mathscr{D}$. For each $y \in D$, let us pick a point $p(y)$ from $\varphi^{-1} y$ and put $A=\{p(y) ; y \in D\}$. Then $A$ is a discrete closed $C$-embedded subset of $X$. Thus $v A=\mathrm{cl}_{v X} A$ is homeomorphic to $\mathrm{cl}_{v Y} D$ under the map $v \varphi$. Thus we have $v \varphi(v X)=v Y$.

(4) $v \varphi$ is an $R C$-map. Let $F$ be regular closed in $v X$ and $E=(v \varphi) F$. Suppose that there is $q \in \mathrm{cl}_{v Y} E-E$. By (2) and the clopenness of $\varphi^{-1} y$, $y \in Y_{d}$, we have $q \notin Y_{d} \cup \mathrm{cl}_{v Y} Y_{e}$. Thus there is $D \in \mathscr{D}$ with $q \in \mathrm{cl}_{v Y} D$ and $\operatorname{cl}_{v Y} D \cap \operatorname{cl}_{v Y} Y_{e}=\varnothing$ by (2). Since $\beta \varphi$ is open by 1.2(5), $v \varphi$ is also * -open and we have that $E \supset(v \varphi)$ int $_{v X} F$ is dense in $\mathrm{cl}_{v Y} E$ because $F$ is regular closed. Let $M=E \cap D \cap Y_{d}$. Then $q \in \operatorname{cl}_{v Y} M$. Let us pick a point $p(y)$ from $\varphi^{-1}(y) \cap F, y \in M . A=\{p(y) ; y \in M\}$ is a discrete closed $C$-embedded subset of $X$ and hence $v A=\mathrm{cl}_{v X} A \subset F$ and $v A$ is homeomorphic to $v M=\mathrm{cl}_{v Y} M$, so $q \in E$ a contradiction.

(5) $v \varphi$ is open $R C$-preserving. Since $v \varphi$ is an $R C$-map, $v \varphi$ is $W Z$ by 1.2(6). Thus the openness of $\beta \varphi$ implies that $v \varphi$ is open by 1.2(1) and $R C$-preserving by $1.2(7)$. 
As a direct consequence of the above theorem, we have the following corollary which is a generalization of the result obtained in [5] if $X$ is realcompact and $\varphi: X \rightarrow Y$ is an open $W Z$ map with $\mathrm{Bd} \varphi^{-1} y=$ compact for each $y \in Y$, then $Y$ is also realcompact.

Corollary 2.7. If $X$ is realcompact and $\varphi: X \rightarrow Y$ is a $\beta$-open map such that $Y_{e}$ is $\varphi-d^{*}$, then $Y$ is also realcompact.

THEOREM 2.8. Let $\varphi: X \rightarrow Y$ and $Z=(\beta \varphi)^{-1} Y_{d} \cup v X$. Then the following are equivalent:

(1) $Z$ is a realcompact and $\varphi$ is a $\beta$-open map such that $Y_{e}$ is $\varphi-d^{*}$.

(2) $\varphi^{\prime}=(\beta \varphi) \mid Z$ is an open perfect map of $Z$ onto $v Y$.

(3) $v \varphi$ is a clopen map of $v X$ onto $v Y$ such that $\operatorname{Bd}(v \varphi)^{-1} q$ is compact for every $q \in v Y$.

(4) $v \varphi$ is a clopen map of $v X$ onto $v Y$ such that $(v Y)_{e}$ is $(v \varphi)-d^{*}$.

Proof. (1) $\Rightarrow$ (2) If $Z=\beta X$, then $\varphi^{\prime}=\beta \varphi$ and $\varphi^{\prime}$ is an open perfect map onto $v Y$. Let $p \in \beta X-Z$ and $q=(\beta \varphi) p$. Then $Z=v Z, \beta Z=\beta X$ and there is $f \in C(\beta X)$ such that $p \in Z(f) \subset \beta X-Z$ and $0 \leq f \leq 1$. Since $\beta \varphi$ is open $W Z$ and $Y_{e}$ is $\varphi-d^{*}$, it is easy to see that $f^{i} \in C(\beta Y)$, $f^{i}(q)=0$ and $f^{i}>0$ on $Y$. Thus $q \in \beta Y-v Y$, so $\varphi^{\prime}$ is a perfect map onto $v Y$. The openness of $\varphi^{\prime}$ follows from 1.2(1,5).

(2) $\Rightarrow$ (3) We shall show that $v \varphi$ is closed. Let $F$ be closed in $v X$ and $q \in \operatorname{cl}_{v Y}(v \varphi) F-(v \varphi) F$. Since $\varphi^{\prime}$ is perfect and every point of $Y_{d}$ is isolated, we have $q \notin Y_{d}$, so $(\beta \varphi)^{-1} q=(v \varphi)^{-1} q$ is disjoint from $\mathrm{cl}_{Z} F$, and hence $q \notin \varphi^{\prime}\left(\mathrm{cl}_{Z} F\right)$, a contradiction. Thus $v \varphi$ is closed. The verifications of other parts are easy. (3) $\Rightarrow(4)$ Evident.

(4) $\Rightarrow(1)$ Since $v \varphi$ is clopen, $\beta(v \varphi)=\beta \varphi$ is open by $1.2(1)$ and hence $\varphi$ is $\beta$-open by 1.2(5). Since $v Y=(v Y)_{e} \cup Y_{d}$, the $(v \varphi)-d^{*}$-ness of $(v Y)_{e}$ $=v Y-Y_{d}$ implies the $\varphi$ - $d^{*}$-ness of $Y_{e}$. Since $Y_{d}=(v Y)_{d}$ and $(v Y)_{e}$ is $(v \varphi)-d^{*}$, we have $Z=(\beta \varphi)^{-1} v Y$, and hence $\varphi^{\prime}: Z \rightarrow v Y$ is an open perfect map which shows that $Z$ is realcompact.

\section{REFERENCES}

[1] L. Gillman and M. Jerison, Rings of Continuous Functions, Van Nostrand, Princeton, N. J., (1960).

[2] Y. Ikeda and M. Kitano, Notes on RC-preserving mappings, Bull. Tokyo Gakugei Univ., Ser. IV, 29 (1977), 53-59.

[3] K. Ikeda, RC-mappings and almost normal spaces, ibid., 50 (1979), 13-17.

[4] T. Ishii, On the completions of maps, Proc. Japan Acad., 50 (1974), 39-43. 
[5] T. Isiwata, Mappings and spaces, Pacific J. Math., 20 (1967), 455-480.

[6] _,$d$-, $d^{*}$-maps and $c b^{*}$-spaces, Bull. Tokyo Gakugei Univ., Ser. IV, 29 (1977), $19-52$.

[7] __ Ultrafilters and mappings, Pacific J. Math., 104 (1983), 371-389.

[8] K. Morita, Completion of hyperspaces of compact subsets and topological completion of open-closed maps, General Topology and Appl., 4 (1974), 217-233.

Received August 18, 1983 and in revised form October 28, 1983.

TOKYO GAKUGEI UNIVERSITY

(184) 4-1-1 NUKUIKITA-MACHI

KOGANEI-SHI, TOKYO, JAPAN 


\section{PACIFIC JOURNAL OF MATHEMATICS EDITORS}

\author{
V. S. VARADARAJAN (Managing Editor) \\ University of California \\ Los Angeles, CA 90024 \\ Charles R. DePrima \\ California Institute of Technology \\ Pasadena, CA 91125 \\ R. FINN \\ Stanford University \\ Stanford, CA 94305
}

\author{
HeRmanN FlaschKa \\ University of Arizona \\ Tucson, AZ 85721 \\ Ramesh A. Gangolli \\ University of Washington \\ Seattle, WA 98195 \\ ROBION KIRBY \\ University of California \\ Berkeley, CA 94720
}

C. C. MOORE

University of California

Berkeley, CA 94720

H. SAMELSON

Stanford University

Stanford, CA 94305

HAROLD STARK

University of California, San Diego

La Jolla, CA 92093

ASSOCIATE EDITORS
R. ARENS
E. F. BECKENBACH
B. H. NeUmanN
F. WOLF
K. YosHIDA (1906-1982)

\section{SUPPORTING INSTITUTIONS}

UNIVERSITY OF ARIZONA

UNIVERSITY OF BRITISH COLUMBIA

CALIFORNIA INSTITUTE OF TECHNOLOGY

UNIVERSITY OF CALIFORNIA

MONTANA STATE UNIVERSITY

UNIVERSITY OF NEVADA, RENO

NEW MEXICO STATE UNIVERSITY

OREGON STATE UNIVERSITY
UNIVERSITY OF OREGON

UNIVERSITY OF SOUTHERN CALIFORNIA

STANFORD UNIVERSITY

UNIVERSITY OF HAWAII

UNIVERSITY OF TOKYO

UNIVERSITY OF UTAH

WASHINGTON STATE UNIVERSITY

UNIVERSITY OF WASHINGTON 


\section{Pacific Journal of Mathematics}

Vol. 119, No. $1 \quad$ May, 1985

Maurice Chacron, Nonisotropic unitary spaces and modules with

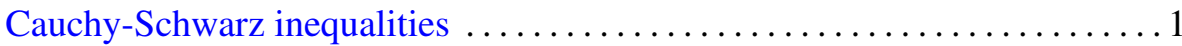

Myriam Dechamps-Gondim, Françoise Piquard and H. Queffélec, On

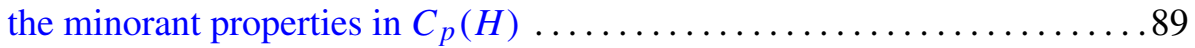

Klaus Floret and V. B. Moscatelli, On bases in strict inductive and projective limits of locally convex spaces .................... 103

Norman Joseph Goldstein, Degenerate secant varieties and a problem on

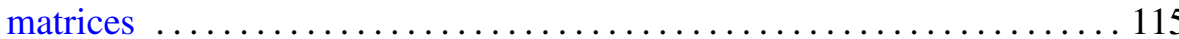

Harold Morris Hastings and Stefan Waner, $G$-bordism with singularities

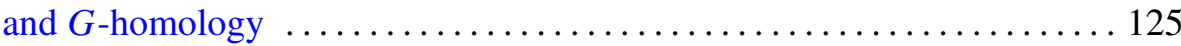

Takesi Isiwata, Clopen realcompactification of a mapping ........... 153

Hisao Kato, Concerning hyperspaces of certain Peano continua and strong

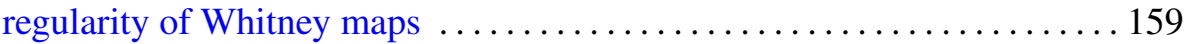

Elyahu Katz and Sidney Allen Morris, Free products of topological groups

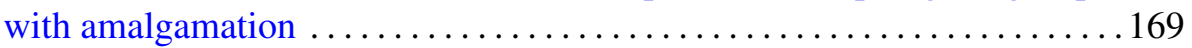

Kyewon Koh Park, Nice dense subsets for ergodic flows and Bernoulli

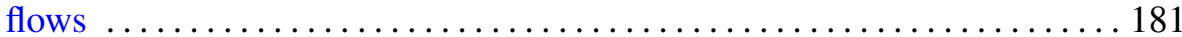

Francis Pastijn and Peter George Trotter, Lattices of completely regular

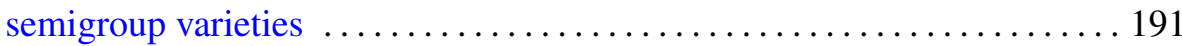

Rae Michael Andrew Shortt, Reticulated sets and the isomorphism of analytic powers

David A. Stegenga and Kenneth R. Stephenson, Generic covering properties for spaces of analytic functions

M. V. Subba Rao and R. Sitaramachandra Rao, On some infinite series of

L. J. Mordell and their analogues 